\title{
Sleep EEG characteristics associated with total sleep time misperception in young adults: an exploratory study
}

\author{
Biyun $\mathrm{Xu}^{1}{ }^{1,2,3^{*}} \mathbb{D}$, Qinghao Cai ${ }^{1}$, Runru Mai ${ }^{1}$, Hailong Liang ${ }^{1}$, Jiayu Huang ${ }^{1}$ and Zhimin Yang ${ }^{1}$
}

\begin{abstract}
Background: Power spectral analysis (PSA) is one of the most commonly-used EEG markers of cortical hyperarousal, and can help to understand subjective-objective sleep discrepancy (SOD). Age is associated with decreased sleep EEG activity; however, the PSA of young adults is currently limited. Thus, this study aimed to examine the correlation of spectral EEG power with total sleep time (TST) misperception in young patients.
\end{abstract}

Methods: Forty-seven young adults were recruited and underwent a polysomnography recording in a sleep laboratory. Clinical records and self-report questionnaires of all patients were collected, and were used to categorize patients into a good sleeper (GS) group $(n=10)$, insomnia with a low mismatch group (IVLM, $n=19)$ or participant with a high mismatch group (IWHM, $\mathrm{n}=18)$. PSA was applied to the first $6 \mathrm{~h}$ of sleep.

Results: IWHM patients exhibited a higher absolute power and relative beta/delta ratio in the frontal region compared to the GS group. No significant difference was observed between the IWLM and GS groups. No significant difference in the above parameters was observed between the IWHM and IWLM groups. Moreover, The SOD of TST was positively correlated with frontal absolute power and the relative beta/delta ratio $(r=0.363, P=0.012 ; r=0.363$, $P=0.012)$, and absolute beta EEG spectral power $(r=0.313, P=0.032)$ as well as the number of arousals.

Conclusions: Increased frontal beta/delta ratio EEG power was found in young patients with a high mismatch but not in those with a low mismatch, compared with good sleepers. This suggests that there exists increased cortical activity in IWHM patients. In addition, the frontal beta/delta ratio and the number of arousals was positively correlated with the SOD of TST.

Keywords: Young adult, Misperception, EEG, Power spectral analysis, Cortical activation

\section{Introduction}

Insomnia is a common disease in modern society with a prevalence rate ranging from 12 to $20 \%$ [1]. Patients with insomnia have a higher risk of mental illness [2] and physical diseases $[3,4]$, which may lead to higher

\footnotetext{
*Correspondence: cloudxby@163.com

${ }^{1}$ Department of Fangcun Sleep-Disorder, the Second Clinical College of Guangzhou University of Chinese Medicine (Guangdong Provincial Hopsital of Chinese Medicine), Guangzhou 510120, China

Full list of author information is available at the end of the article
}

healthcare and medical costs, higher chances of absenteeism, traffic accidents, falling and a poor quality-of-life [4-7].

Subjective-objective sleep discrepancy (SOD), also called sleep misperception, refers to the underestimation of total sleep time (TST) and overestimation of sleep onset latency (SOL). The SOD of TST is obtained by subtracting self-reported TST (sTST) values from objective TST (oTST) values detected by polysomnography (PSG) [8]. SOD is very common in insomnia patients $[9,10]$ and is extremely common in paradoxical insomnia which was listed in the International Classification of Sleep original author(s) and the source, provide a link to the Creative Commons licence, and indicate if changes were made. The images or other third party material in this article are included in the article's Creative Commons licence, unless indicated otherwise in a credit line to the material. If material is not included in the article's Creative Commons licence and your intended use is not permitted by statutory regulation or exceeds the permitted use, you will need to obtain permission directly from the copyright holder. To view a copy of this licence, visit http://creativecommons.org/licenses/by/4.0/. The Creative Commons Public Domain Dedication waiver (http://creativeco mmons.org/publicdomain/zero/1.0/) applies to the data made available in this article, unless otherwise stated in a credit line to the data. 
Disorders-2nd Edition (ICSD-2), but cancelled in ICSD-3 mainly due to a lack of consensus on its precise definition. Although it was cancelled, the symptom is worth studying [11] since it is an important aspect for understanding the mechanism of insomnia.

Several studies have attempted to explain the possible mechanisms of SOD in insomnias and have accumulated 13 possible mechanisms thereof that are supported by good-quality evidence [9]. One of these potential mechanisms is related to cortical hyperarousal. Power spectral analysis (PSA) is one of the most commonly-used EEG markers of cortical hyperarousal [12]. PSA can show whether information processing is in an enhanced state, which may cause the misperception of sleeping state [13]. The power of each waveform is defined as the area under the waveform, where a high amplitude represents high power [9].

Previous studies have shown that patients with primary insomnia (PI) have more high-frequency EEG activity, especially beta-band activity, during non-rapid eye movement (NREM) sleep [14-18] than healthy controls. This finding is also consistent with the hyperarousal theory of insomnia. However, there is still no consensus on other power bands during NREM sleep. For example, a metaanalysis including 532 patients with insomnia disorder (ID) and 445 good sleepers performed by Zhao et al. [18] found that patients with ID exhibited increased theta, alpha, and sigma power during NREM sleep. Riedner et al. [17] found that ID patients exhibited no difference in slow wave (specifically $<5 \mathrm{~Hz}$ ) and sigma (spindle) frequencies (specifically $11-16 \mathrm{~Hz}$ ) compared with GS. This difference could be explained by a number of reasons, one of which is the difference in various subtypes of insomnia. Many of the diseases studied fall under the umbrella term of PI, which is a broad term that includes paradoxical insomnia (ParI) and psychophysiological insomnia (PsyI), as well as idiopathic insomnia. However, some subtypes, for example [9] individuals with and without sleep misperception, are not considered in most studies. Therefore, it has been suggested that future studies on EEG spectral features should patients from different insomnia subtypes. To our knowledge, only several studies aimed to investigate SOD to understand the underlying mechanism of insomnia. Krystal et al. [19], St-Jean et al. [20] and Lecci et al. [21] categorized their patients according to SOD, but there was no consistency in the spectral power analyses in these studies and only elderly patients ( $40-80$ years old vs. $40.21+9.38$ vs $40-85$ years old) were included. Krystal et al. [19] reported that lower delta and greater alpha, sigma and beta NREM EEG activity was found in patients with subjective insomnia but not in those with objective insomnia, compared with normal subjects. St-Jean et al. [20] reported that patients with ParI exhibited higher absolute delta activity at the standardized F3, C3, and P3 electrodes compared to those with PsyI.

It has also shown that there is a relationship between age and s decrease of sleep EEG activity [22, 23]. Compared to young individuals, elderly patients exhibit less N3 and spindle activity during non-rapid eye movement (NREM) sleep, and a smaller proportion of rapid eye movement (REM) sleep [24]. It has also been shown that absolute power in the delta, theta and sigma bands decline with age for both females and males [23].

Table 1 Demographic characteristics of all participants

\begin{tabular}{|c|c|c|c|c|}
\hline Variables & $G S(n=10)$ & IWHM $(n=18)$ & IWLM $(n=19)$ & Statistics \\
\hline Age, years & $26.0[25.0,32.0]$ & $30.5[25.8,38.2]$ & $25.0[23.0,38.0]$ & $\mathrm{H}=3.378, p=0.185$ \\
\hline $\operatorname{Sex}(F / M)$ & $5 / 5$ & 9/9 & $16 / 3$ & $x^{2}=5.616, p=0.060$ \\
\hline Race & & & & $x^{2}=0.000, p=1.000$ \\
\hline Han & 10 & 18 & 19 & \\
\hline Non-Han & 0 & 0 & 0 & \\
\hline Place of residence & & & & $x^{2}=1.233, p=0.540$ \\
\hline Downtown & 9 & 13 & 15 & \\
\hline Suburb & 1 & 4 & 3 & \\
\hline Village & 0 & 1 & 1 & \\
\hline Marriage & & & & $x^{2}=2.798, p=0.247$ \\
\hline Unmarried & 7 & 7 & 10 & \\
\hline Married & 3 & 10 & 9 & \\
\hline Bereavement/divorce & 0 & 1 & 0 & \\
\hline Family history of insomnia (Y/N) & $1 / 9$ & $3 / 15$ & $1 / 18$ & $x^{2}=1.243, p=0.537$ \\
\hline Family history of psychosis $(\mathrm{Y} / \mathrm{N})$ & $0 / 10$ & $0 / 18$ & $1 / 18$ & $x^{2}=1.474, p=0.479$ \\
\hline
\end{tabular}

GS: good sleeper; IWLM: insomnias with a low mismatch; IWHM: insomnias with a high mismatch 
Table 2 PSQI scores, SCL90 scores, and PSG characteristics of all participants

\begin{tabular}{|c|c|c|c|c|}
\hline Variable & $G S(n=10)$ & $\operatorname{IWHM}(n=18)$ & $\operatorname{IWLM}(n=19)$ & Statistics \\
\hline PSQI total score & $3.5[2.0,6.2]$ & $13.5[11.5,16.3]^{\mathrm{a}}$ & $11.0[9.0,14.0]^{\mathrm{a}}$ & $H=18.882, P<0.001$ \\
\hline SCL-90 total score & $109.5[103.5,129.3]$ & $174.5[155.8,223.8]^{\mathrm{a}}$ & $148.0[127.5,180.0]^{\mathrm{a}}$ & $H=16.037, P<0.001$ \\
\hline TST (min) & $394.25 \pm 45.25$ & $415.06 \pm 40.20$ & $381.95 \pm 39.85$ & $F=3.026, P=0.059$ \\
\hline $\mathrm{SPT}$ (min) & $418.60 \pm 47.39$ & $459.03 \pm 42.65$ & $431.84 \pm 49.77$ & $F=2.837, P=0.069$ \\
\hline $\mathrm{SE}, \%$ & $89.27 \pm 3.92$ & $87.84 \pm 7.00$ & $86.24 \pm 8.39$ & $F=0.626, P=0.540$ \\
\hline $\mathrm{SOL}(\min )$ & $11.50[7.13,23.38]$ & $8.25[4.00,12.63]$ & $5.50[3.00,10.00]$ & $H=2.697, P=0.260$ \\
\hline \%NREM stage 1 & $5.00[3.00,6.25]$ & $5.00[4.00,8.50]$ & $4.00[3.00,7.00]$ & $H=1.158, P=0.560$ \\
\hline \%NREM stage2 & $59.40 \pm 8.51$ & $61.17 \pm 8.61$ & $62.16 \pm 7.63$ & $F=0.371, P=0.692$ \\
\hline$\%$ SWS & $13.60 \pm 4.79$ & $10.06 \pm 6.49$ & $12.37 \pm 4.82$ & $F=1.528, P=0.228$ \\
\hline \%REM & $21.90 \pm 6.61$ & $22.61 \pm 3.27$ & $19.31 \pm 5.14$ & $F=2.259, P=0.116$ \\
\hline Number of awakenings & $23.50[19.50,29.50]$ & $24.00[16.00,31.00]$ & $19.00[13.00,27.00]$ & $\mathrm{H}=2.147, P=0.342$ \\
\hline Number of arousals & $17.00[8.25,20.50]$ & $24.00[12.75,47.50]$ & $26.00[17.00,47.00]$ & $H=3.730, P=0.155$ \\
\hline Arousal index & $2.69[1.22,3.21]$ & $3.71[1.77,7.42]$ & $4.17[2.28,7.55]$ & $H=3.611, P=0.164$ \\
\hline Number of arousals of NREM & $15.00[8.25,20.50]$ & $19.50[10.00,46.50]$ & $22.00[17.00,41.00]$ & $H=3.233, P=0.199$ \\
\hline Number of arousals of REM & $0.00[0.00,3.00]$ & $1.00[0.00,3.25]$ & $1.00[0.00,5.00]$ & $H=1.339, P=0.512$ \\
\hline
\end{tabular}

GS: good sleeper; IWLM: insomnias with a low mismatch; IWHM: insomnias with a high mismatch

PSQI: Pittsburgh Sleep Quality Index; TST: total sleep time; SPT: sleep period time; SE: sleep efficiency; SOL: sleep onset latency; NREM: non-rapid eye movement; SWS: slow wave sleep; REM: rapid eye movement

The statistical value $\mathrm{H}$ represents the use of a Kruskal-Wallis non-parametric analysis among three group using a Bonferroni correction for post-hoc analysis, while the statistical value $F$ represents the use of a one-way ANOVA using the least significant difference test for post-hoc analysis

${ }^{a} P<0.05$ versus GS. There was no difference between the IWHW and IWLW groups

Table 3 Comparison of absolute EEG spectral power among the experimental groups $\left(\mu v^{2}\right)$

\begin{tabular}{|c|c|c|c|c|}
\hline Variable & $\mathrm{GS}(\mathrm{n}=10)$ & IWHM (n=18) & IWLM (n= 19) & Statistics \\
\hline \multicolumn{5}{|l|}{ Frontal derivation } \\
\hline Delta $(1-4 \mathrm{~Hz})$ & $46.47 \pm 21.05$ & $37.61 \pm 15.30$ & $46.65 \pm 25.95$ & $\mathrm{~F}=0.981, P=0.383$ \\
\hline Theta $(4-8 \mathrm{~Hz}$ & $2.90[2.29,4.22]$ & $3.23[2.67,3.99]$ & $4.91[2.59,5.83]$ & $H=3.481, P=0.175$ \\
\hline Alpha $(8-12 \mathrm{~Hz})$ & $1.87[1.16,3.37]$ & $2.53[1.55,2.99]$ & $2.78[1.97,3.89]$ & $H=2.693, P=0.260$ \\
\hline Sigma $(12-16 \mathrm{~Hz})$ & $0.80[0.55,1.27]$ & $1.09[0.69,1.56]$ & $1.40[0.88,1.76]$ & $H=4.137, P=0.126$ \\
\hline Beta $(16-32 \mathrm{~Hz})$ & $0.71[0.57,0.99]$ & $1.08[0.69,1.80]$ & $1.06[0.79,1.50]$ & $H=4.062, P=0.131$ \\
\hline Beta /Delta & $0.01[0.01,0.03]$ & $0.03[0.02,0.04]^{a}$ & $0.03[0.02,0.04]$ & $H=6.904, P=0.032$ \\
\hline Beta/Theta & $0.27[0.17,0.37]$ & $0.34[0.23,0.53]$ & $0.25[0.18,0.36]$ & $H=1.907, P=0.385$ \\
\hline Alpha/Delta & $0.04[0.03,0.08]$ & $0.07[0.04,0.09]$ & $0.07[0.05,0.11]$ & $H=3.086, P=0.214$ \\
\hline Alpha/Theta & $64.00 \pm 16.43$ & $76.60 \pm 41.44$ & $74.33 \pm 30.73$ & $F=0.487, P=0.618$ \\
\hline \multicolumn{5}{|l|}{ Central derivation } \\
\hline Delta $(1-4 \mathrm{~Hz})$ & $37.99 \pm 12.36$ & $33.89 \pm 19.01$ & $46.48 \pm 20.94$ & $\mathrm{~F}=2.154, P=0.128$ \\
\hline Theta $(4-8 \mathrm{~Hz}$ & $4.77[3.37,4.99]$ & $4.11[3.21,4.63]$ & $4.93[2.98,8.85]$ & $\mathrm{H}=2.872, P=0.238$ \\
\hline Alpha $(8-12 \mathrm{~Hz})$ & $2.51[1.66,3.24]$ & $2.79[1.89,3.52]$ & $2.70[1.83,4.02]$ & $H=0.710, P=0.701$ \\
\hline Sigma $(12-16 \mathrm{~Hz})$ & $1.43[1.04,1.84]$ & $1.66[1.21,2.76]$ & $1.75[1.37,2.51]$ & $H=2.625, P=0.269$ \\
\hline Beta $(16-32 \mathrm{~Hz})$ & $0.81[0.66,1.25]$ & $1.29[0.87,1.62]$ & $1.16[0.72,1.65]$ & $H=2.139, P=0.343$ \\
\hline Beta/Delta & $0.02[0.02,0.04]$ & $0.04[0.02,0.06]$ & $0.03[0.02,0.04]$ & $H=3.087, P=0.214$ \\
\hline Beta/Theta & $0.18[015,0.31]$ & $0.28[0.20,0.43]$ & $0.20[0.15,0.35]$ & $H=2.385, P=0.303$ \\
\hline Alpha/Delta & $0.07[0.04,0.09]$ & $0.08[0.05,0.14]$ & $0.06[0.05,0.09]$ & $H=2.632, P=0.268$ \\
\hline Alpha/Theta & $0.57[0.47,0.66]$ & $0.58[0.51,0.92]$ & $0.52[0.42,0.75]$ & $H=1.774, P=0.412$ \\
\hline
\end{tabular}

GS: good sleeper; IWLM: insomnias with a low mismatch; IWHM: insomnias with a high mismatch. The statistical value $\mathrm{H}$ represents the use of a Kruskal-Wallis nonparametric analysis among three group using a Bonferroni correction for post-hoc analysis, while the statistical value $\mathrm{F}$ represents the use of a one-way ANOVA using the least significant difference test for post-hoc analysis

${ }^{\text {a }} P<0.05$ versus GS. There was no difference between the IWHW and IWLW groups 


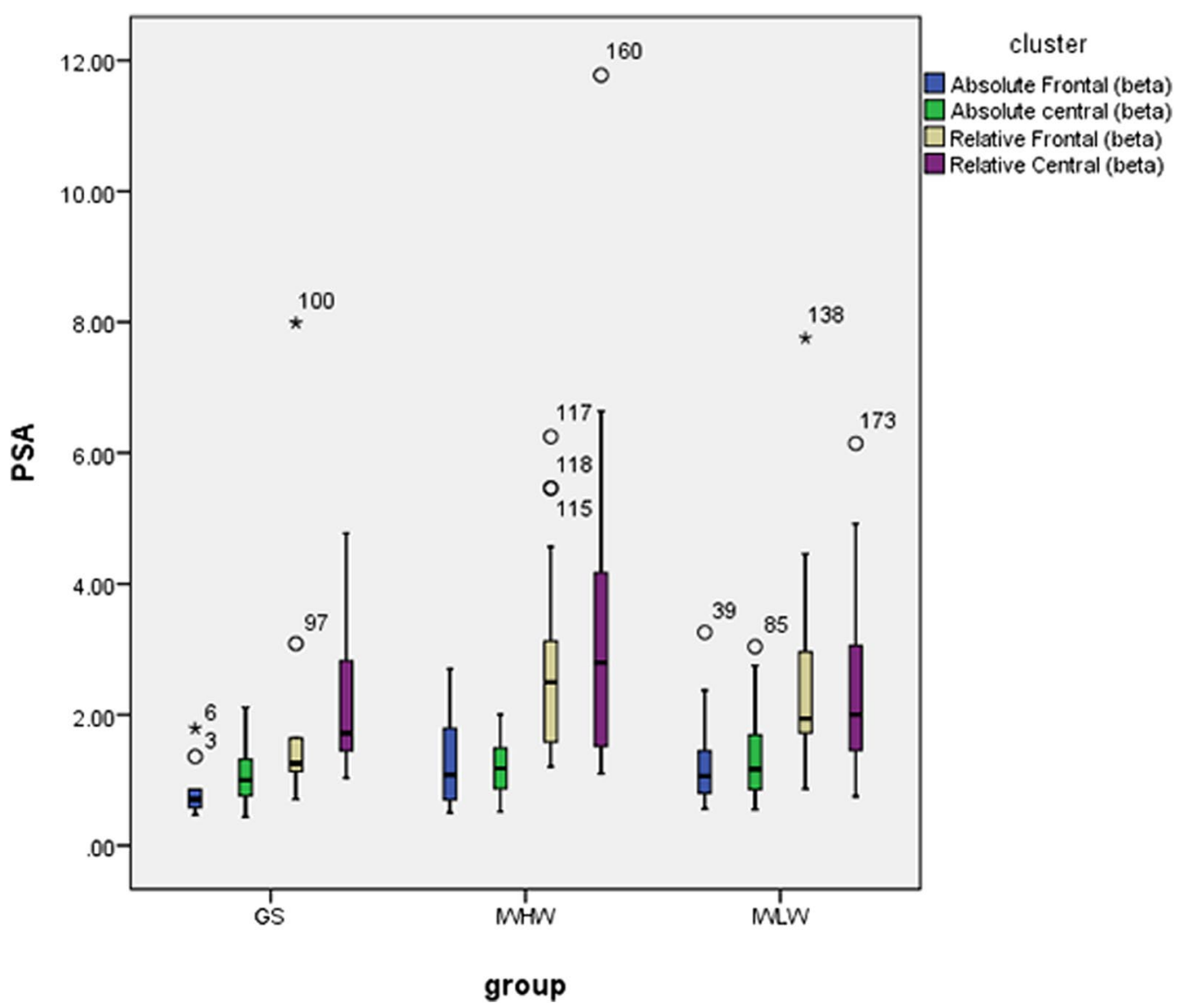

Fig. 1 Absolute and relative beta in the frontal and central region. GS: good sleeper; IWLM: insomnias with a low mismatch; IWHM: insomnias with a high mismatch

Importantly, previous research has failed to combine age data with insomnia subtype information to study the PSA of SOD.

In this study, we aimed to compare the PSA of three groups of young patients: (1) patients who overestimated their total sleep time by at least $2 \mathrm{~h}$; (2) patients who correctly estimated their sleep; (3): good sleepers (GS). Our findings may be important to clinical and public health as well as the treatment and management of insomnia [9].

\section{Materials and methods Participants}

Seventy participants aged between 18 and 40 years-old were recruited from the Guangdong Provincial Hospital of Chinese Medicine through posters from May 2016 to November 2017. All subjects were asked to complete a two-week sleep diary followed by a single all-night PSG recording in a sleep laboratory. Personal information was obtained from all subjects, including age, sex, race, place of residence, marital status, family history of insomnia and psychosis. Two self-reported questionnaires, the Pittsburgh Sleep Quality Index (PSQI) [25] and the Symptom Checklist 90 (SCL-90), were given to each participant. Subjective sleep quality was determined by selfreported TST after PSG. The subjects were asked two questions about their perceived sleep within $2 \mathrm{~h}$ after PSG completion: (1) "How long did you sleep last night?" and (2) "Did you sleep as usual?". In this way, the sTST of the patient was obtained. For example, if the patient replied that they slept for $6 \mathrm{~h}$ during the previous night, $360 \mathrm{~min}$ was his/her sTST.

Subjects were categorized as GS according to the following criteria: (1) reported no difficulty in sleep according to the two-week sleep diary (i.e. sleep onset $(\mathrm{SO})<30 \mathrm{~min}$, wake after sleep onset (WASO) $<40 \mathrm{~min}$, TST between 6.0 and $8.0 \mathrm{~h}$, or sleep efficiency $(\mathrm{SE}) \geq 85 \%$ ); (2) had a PSQI score $<7$ [25], SE $>85 \%$ or TST $>6$ h.

Participants were categorized as insomnia patients if they met the following criteria: (1) diagnosed with chronic insomnia disorder (International Classification of Sleep Disorders, 3rd edition); (2) reported at least 


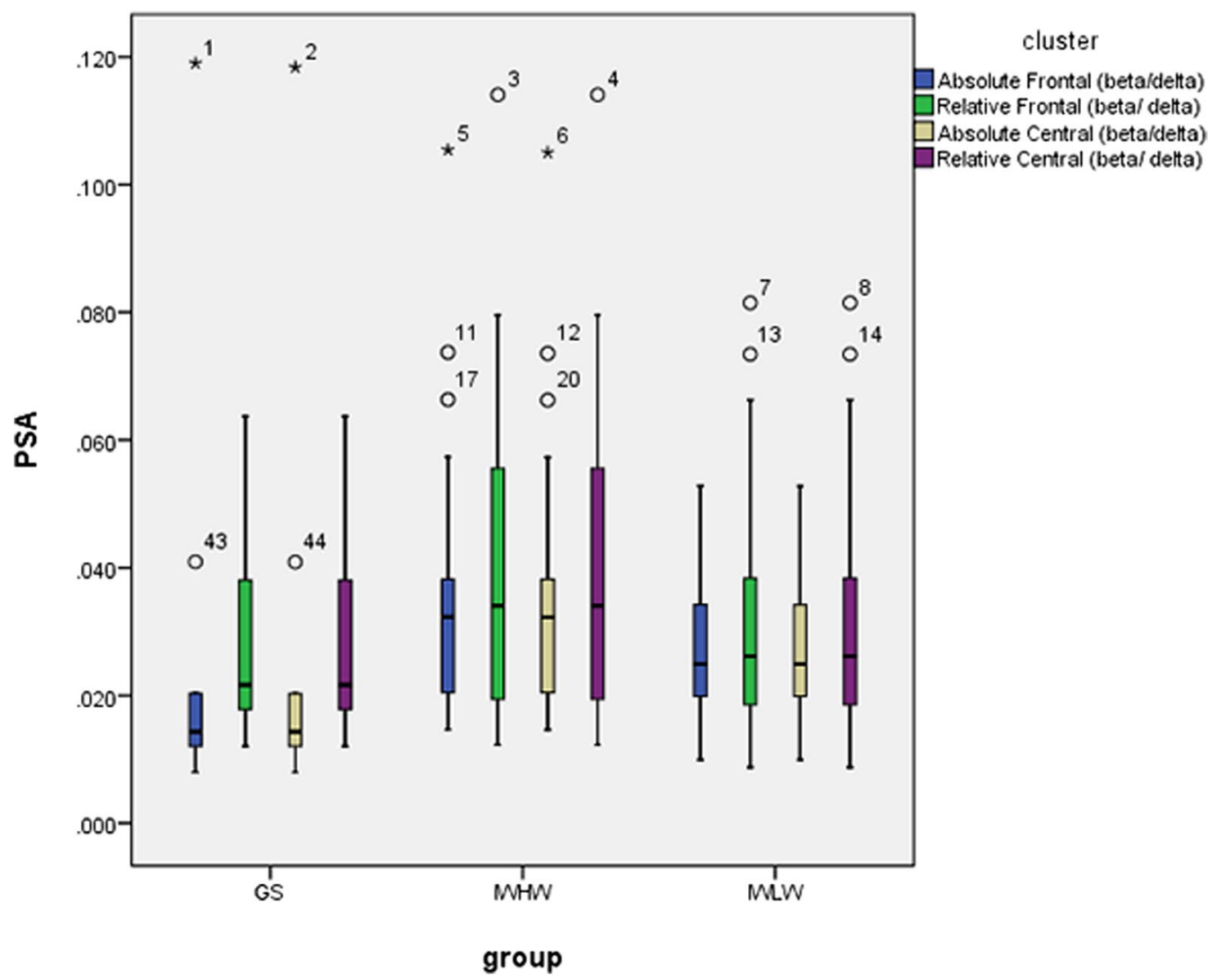

Fig. 2 Absolute power and relative beta/delta in the frontal and central regions. GS: good sleeper; IWLM: insomnias with a low mismatch; IWHM: insomnias with a high mismatch

three nights per week of sleep difficulty (i.e.SO $>30 \mathrm{~min}$, WASO $>40 \mathrm{~min}$, sTST $<6.0 \mathrm{~h}$, or $\mathrm{SE}<85 \%$ ); (3) had a PSQI score of $>7$; (4) had difficulty sleeping for more than 3 months; (5) did not have other medical, psychological, or sleeping disorders and did not take any medications that would affect sleep (e.g. sedative and hypnotic drugs, antidepressants, anti-schizophrenia drugs, etc.).

Insomnia patients were further categorized into two subgroups based on their SOD of TST. These two subgroups comprised patients with low mismatch (IWLM) and patients with high mismatch (IWHM). The SOD of TST was operationalized as the values of the differences between subjective and objective measures (i.e. sTSToTST value) [8]. IWLM patients were those individuals who met the criteria of chronic insomnia disorder and had an $\mathrm{SOD}<60 \mathrm{~min}$ in TST. IWHM were defined as patients who met the criteria of chronic insomnia disorder and had normal PSG parameters (i.e. SE $>85 \%$ and TST $>6.5 \mathrm{~h}$ ) and SOD $>120 \mathrm{~min}$ in TST. In both the IWHM and IWLM subgroups, patients were excluded if they met one of the following criteria: (1) diagnosed with another Axis I disorder or any other sleeping disorder (e.g. idiopathic insomnia, sleep apnea, which was defined as an apnea-hypopnea index of more than five events per hour using PSG, or restless leg syndrome); (2) affected by other external factors that might affect insomnia (e.g. physical pain caused by medical diseases, drugs affecting sleeping structure, alcohol consumption, other treatments, etc.); (3) go to sleep later than 0:00 am or wake up before 6:00 am, or had irregular sleeping schedules.

Based on the inclusion and exclusion criteria, 47 participants were included in the study: GS group $(n=10$; 5 males, 5 females), IWHM group $(n=18 ; 9$ males, 9 females), and IWLM group ( $\mathrm{n}=19 ; 3$ males, 16 females).

\section{PSQI and SCL-90}

The PSQI is a questionnaire consisting of 21 items and has been commonly used to evaluate subjective sleep quality. The higher the score, the greater the severity of insomnia. A score $>7$ indicates abnormal sleeping (severe difficulty in at least two areas or moderate difficulty in more than three areas).

The SCL-90 is one of the most widely used mental health scales in the field of psychiatry. It is a 90 -item, selfreported symptom inventory. The score for each item is summed, yielding a total score that covers ten aspects. 
Table 4 Comparison of relative EEG spectral power among the experimental groups

\begin{tabular}{|c|c|c|c|c|}
\hline Variable & $G S(n=10)$ & IWHM $(n=18)$ & $\operatorname{IWLM}(n=19)$ & Statistics \\
\hline \multicolumn{5}{|l|}{ Frontal derivation } \\
\hline Delta $(1-4 \mathrm{~Hz})$ & $87.96[79.34,90.48]$ & $80.74[75.23,83.99]$ & $80.81[76.26,85.81]$ & $H=5.046, P=0.080$ \\
\hline Theta $(4-8 \mathrm{~Hz}$ & $5.85[4.54,8.54]$ & $7.39[6.24,9.56]$ & $7.20[6.61,10.78]$ & $\mathrm{H}=3.820, P=0.148$ \\
\hline Alpha $(8-12 \mathrm{~Hz})$ & $3.77[2.49,6.48]$ & $5.08[3.56,6.84]$ & $5.10[3.80,8.26]$ & $H=2.767, P=0.251$ \\
\hline Sigma $(12-16 \mathrm{~Hz})$ & $1.31[1.16,2.71]$ & $2.51[1.75,3.35]$ & $2.15[1.62,4.32]$ & $H=3.498, P=0.174$ \\
\hline Beta $(16-32 \mathrm{~Hz})$ & $1.26[1.09,2.00]$ & $2.50[1.54,3.49]$ & $1.94[1.65,2.99]$ & $H=5.756, P=0.056$ \\
\hline Beta /Delta & $0.01[0.01,0.03]$ & $0.03[0.02,0.04]^{\mathrm{a}}$ & $0.03[0.02,0.04]$ & $H=6.904, P=0.032$ \\
\hline Beta/Theta & $0.27[0.17,0.37]$ & $0.34[0.23,0.53]$ & $0.25[0.18,0.36]$ & $H=1.907, P=0.385$ \\
\hline Alpha/Delta & $0.04[0.03,0.08]$ & $0.07[0.04,0.09]$ & $0.07[0.04,0.11]$ & $H=3.183, P=0.204$ \\
\hline Alpha/Theta & $64.00 \pm 16.42$ & $76.60 \pm 41.44$ & $74.32 \pm 30.73$ & $\mathrm{~F}=0.487, P=0.618$ \\
\hline \multicolumn{5}{|l|}{ Central derivation } \\
\hline Delta $(1-4 \mathrm{~Hz})$ & $78.42[74.84,86.28]$ & $74.67[66.94,82.17]$ & $77.34[74.20,82.11]$ & $H=3.930, P=0.140$ \\
\hline Theta $(4-8 \mathrm{~Hz}$ & $10.04[6.57,10.67]$ & $9.98[8.57,11.37]$ & $9.54[8.07,12.22]$ & $H=0.281, P=0.869$ \\
\hline Alpha $(8-12 \mathrm{~Hz})$ & $5.74[3.51,7.13]$ & $5.94[4.59,9.21]$ & $4.84[4.01,7.00]$ & $H=2.483, P=0.289$ \\
\hline Sigma $(12-16 \mathrm{~Hz})$ & $3.11[2.35,4.39]$ & $4.39[3.15,6.77]$ & $3.29[2.62,4.55]$ & $H=5.447, P=0.066$ \\
\hline Beta $(16-32 \mathrm{~Hz})$ & $1.72[1.43,3.05]$ & $2.79[1.51,4.42]^{\mathrm{a}}$ & $2.00[1.45,3.38]$ & $H=3.121, P=0.210$ \\
\hline Beta /Delta & $0.02[0.02,0.04]$ & $0.04[0.02,0.06]$ & $0.03[0.02,0.04]$ & $H=3.087, P=0.214$ \\
\hline Beta/Theta & $0.18[0.15,0.31]$ & $0.28[0.20,0.43]$ & $0.20[0.15,0.35]$ & $H=2.385, P=0.303$ \\
\hline Alpha/Delta & $0.07[0.04,0.09]$ & $0.08[0.05,0.14]$ & $0.06[0.05,0.09]$ & $H=2.632, P=0.268$ \\
\hline Alpha/Theta & $0.57[0.47,0.66]$ & $0.58[0.51,0.92]$ & $0.52[0.42,0.75]$ & $H=1.774, P=0.412$ \\
\hline
\end{tabular}

GS: good sleeper; IWLM: insomnias with a low mismatch; IWHM: insomnias with a high mismatch

The statistical value $\mathrm{H}$ represents the use of a Kruskal-Wallis non-parametric analysis among three group using a Bonferroni correction for post-hoc analysis, while the statistical value $\mathrm{F}$ represents the use of a one-way ANOVA using the least significant difference test for post-hoc analysis. ${ }^{a} P<0.05$ versus GS. There was no difference between the IWHW and IWLW groups

The higher the total score, the greater the risk of developing psychological distress [26].

\section{PSG recordings}

In conventional PSG (Nicolet, ONE, EEG 32, USA), the international 10-20 system was used to record EEG. In this study, the grounding electrode was placed on the frontal pole midline point and the bilateral ear electrodes were used as the reference. All electrographic electrodes were placed according to the AASM 2.6 recommended guidelines. The impedance was kept below $5 \mathrm{k} \Omega$ for all electrodes. The surface electrodes included six EEG (two central electrodes [C3, C4], two frontal EEG electrodes [F3, F4], and two occipital EEG electrode [O1, O2)]), two electro-oculogram (E1, E2), submental electromyogram (EMG: Chin1-Chin2), electrocardiogram (ECG), and two reference electrodes (A1, A2). In addition, tibialis EMG and respiration were used to exclude periodic limb movements (a PLMSI > 15) and sleep apnea (an apnea-hypopnea index $>5$ ), respectively. Participants were asked to sleep at their usual time (before 0:00 am) and wake up at 7:00 am. The sampling rate of EEG was $500 \mathrm{~Hz}$ and the filter settings were as follows: notch frequency at $60 \mathrm{~Hz}$; low pass filter at $35 \mathrm{~Hz}$; high pass filter at $0.3 \mathrm{~Hz}$.
Sleep records were reviewed and scored by a registered PSG technician according to the revised AASM 2.5 sleeping scoring criteria [27]. The sleeping continuity parameters, including TST, SPT, SE (ratio of TST to time in bed $\times 100 \%$ ), and SOL, and sleeping architecture parameters, including the number of awakenings, the number of arousals, arousal index, percentage of NREM stage 1 and 2, slow wave sleep (SWS) or NREM stage 3, and REM sleep of TST were analyzed.

\section{Spectral analysis}

Normal sleep time is 6.0 to $8.0 \mathrm{~h}$, and therefore we analyzed the first $6 \mathrm{~h}$ of the PSG recordings. The data from the central and frontal EEG electrode (averaged C3-A2 and C4-A1 channels, averaged F3-A2 and F4-A1 channels) were generated using software of Nicolet EEG band width tools.

Most of the common artifacts were due to improper click placements (such as electrode popping, ECG or pulse artifact), body movement (muscle artifact, eye movement artifact or major body movement) or environmental factors (overheated which lead to slow-frequency artifacts). We optimized the mastoid electrodes so that ECG and pulse artifacts could be minimized. Secondly, 
Table 5 Correlation between absolute EEG spectral power and SOD of TST

\begin{tabular}{lcc}
\hline Variables & SOD of TST & \\
\cline { 2 - 3 } & $\mathbf{r}$ & $\boldsymbol{P}$ \\
\hline Frontal derivation & & \\
Delta (1-4 Hz) & -0.202 & 0.173 \\
Theta (4-8 Hz) & -0.032 & 0.833 \\
Alpha (8-12 Hz) & 0.058 & 0.698 \\
Sigma (12-16 Hz) & -0.013 & 0.931 \\
Beta (16-32 Hz) & 0.200 & 0.179 \\
Beta/Delta & 0.363 & 0.012 \\
Beta/Theta & 0.208 & 0.161 \\
Alpha/Delta & 0.171 & 0.249 \\
Alpha/Theta & 0.182 & 0.221 \\
Central derivation & & \\
Delta (1-4 Hz) & -0.109 & 0.467 \\
Theta (4-8 Hz & -0.063 & 0.673 \\
Alpha (8-12 Hz) & 0.188 & 0.205 \\
Sigma(12-16 Hz) & 0.156 & 0.295 \\
Beta (16-32 Hz) & 0.216 & 0.145 \\
Beta/Delta & 0.249 & 0.091 \\
Beta/Theta & 0.188 & 0.256 \\
Alpha/Delta & 0.256 & 0.082 \\
Alpha/Theta & 0.169 & 0.257 \\
\hline SOD: Subjective-objective sleep discrepancy: TST:total sleep time &
\end{tabular}

we kept impedance below $5 \mathrm{k} \Omega$ to avoid electrode popping. At the same time, we maintained a temperature of $20{ }^{\circ} \mathrm{C}$ in the sleep laboratory which is the standard setting to ensure that the subjects completed the test in a comfortable environment, and avoided the influence of slow-frequency artifacts from sweat. A notch filter at $50 \mathrm{~Hz}$ was applied to avoid power line contamination of the electrical signals. Then, we set a high frequency filter to $35 \mathrm{~Hz}$ to reduce most of the interference from EMG. We chose this cutoff values as the frequency of EMG activity signal is generally contained in higher frequency bands and since the AASM recommend that EMG low frequency and high frequency filter cutoffs should be at $10 \mathrm{~Hz}$ and $100 \mathrm{~Hz}$, respectively, to capture the muscle activity. Finally, the data fragments that were displaced or cut off due to movements or that were obviously different from the background were to excluded by visual inspection (e.g. due to the excessive loss of occipital EEG electrode signal, these data were not included in this study). Therefore, artifacts in each recording were visually inspected and removed accordingly.

The beta $(16-32 \mathrm{~Hz})$, sigma $(12-16 \mathrm{~Hz})$, alpha $(8-12 \mathrm{~Hz})$, delta $(0.5-4 \mathrm{~Hz})$, and theta $(4-8 \mathrm{~Hz})$ band activity was extracted for PSA analysis. The values of relative spectral power were calculated by dividing the absolute power of each frequency band by the power of the total power spectrum.

\section{Statistical analysis}

Statistical analysis was performed using the SPSS software (ver. 24.0) and with an unpaired two-tailed test of significance. A normality test and Levene's test were used to check whether the data followed a normal distribution. A Chi-square test was used for demographic characteristics except for age. Normally distributed data with homogeneous variance were compared using a one-way ANOVA, while others were compared using a non-parametric analysis (Kruskal-wallis) with post-hoc analysis. The statistical value $\mathrm{H}$ represents the use of non-parametric analysis, while the statistical value $\mathrm{F}$ represents the use of a one-way ANOVA. In addition, we used pairwise least significant difference post-hoc tests after a oneway ANOVAs and a Bonferroni correction for multiple comparison after a Kruskal-Wallis test. Spearman's or Pearson's correlation analysis was used to determine the correlation between the EEG spectral power (absolute and relative) and the SOD of TST (after data normality was confirmed). A $P$-value $<0.05$ was considered statistically significant.

\section{Results}

Baseline characteristics

There was no significant differences in age, sex, race, place of residence, marital status, family history of insomnia, or family history of psychosis among the three groups (Table 1).

\section{PSQI, SCL90, and PSG characteristics}

The comparisons of the PSQI score, SCL-90 score, and PSG among the three groups are shown in Table 2. The IWHM and IWLM groups showed higher PSQI and SCL-90 scores compared to the GS group. However, there was no significant difference in the PSQI or SCL-90 scores between the IWHM and IWLM groups. The PSG parameters were not significantly different among the GS, IWHM, and IWLM groups.

\section{Absolute EEG spectral power}

Post-hoc analysis (Bonferroni correction) revealed that the IWHM group exhibited a significantly higher frontal beta/delta ratio than the GS group. No significant difference was observed between the IWLM and GS groups. There was no significant difference in these parameters between the IWHM and IWLM groups (Table 3, Figs. 1, 2). 


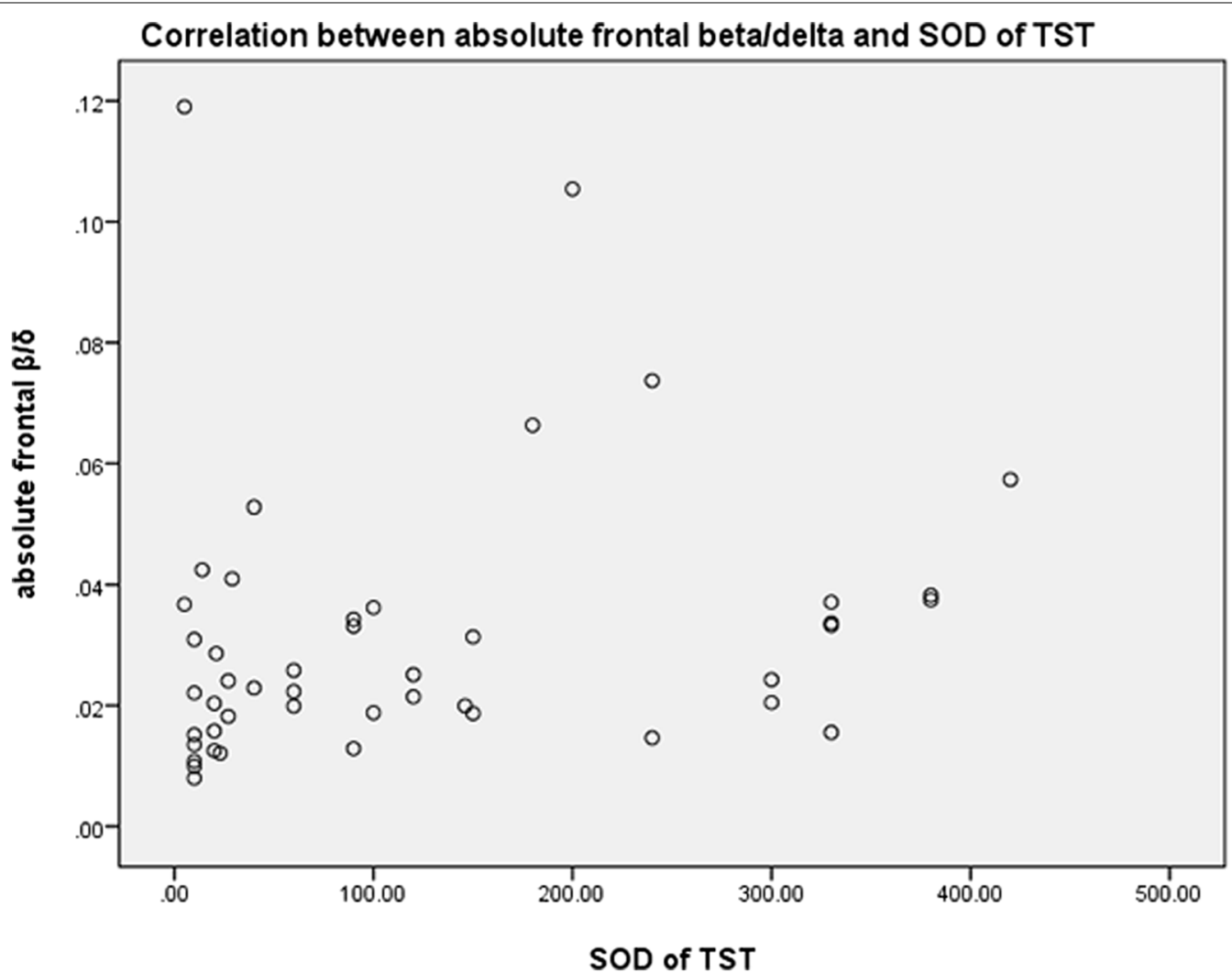

Fig. 3 Correlation between absolute frontal beta/dela spectral power and SOD of TST. SOD: Subjective-objective sleep discrepancy; TST: total sleep time

\section{Relative EEG spectral power}

The average NREM activity for the beta/delta ratio in the frontal area was significantly different among the three groups. Post-hoc analysis (Bonferroni correction) showed that the frontal beta/delta ratio in the IWHM group was higher than that in the GS group. No significant difference was observed between the IWLM and GS groups and no significant difference in relative spectral power was observed between the IWHM and IWLM groups (Table 4, Figs. 1, 2).

Correlation between absolute EEG spectral power and SOD Spearman's correlation was performed on the SOD due to the non-normality of the data. The SOD of TST was positively correlated with absolute frontal beta/delta ratio ( $\mathrm{r}=0.363, P=0.012$ ) (Table 5 , Fig. 3 ).

\section{Correlation between relative EEG spectral power and SOD}

Spearman's correlation was performed on the SOD due to the non-normality of the data. The SOD of TST was positively correlated with relative frontal beta/delta ratio $(r=0.363, P=0.012)$ and the absolute beta EEG spectral power $(\mathrm{r}=0.313, P=0.032)$ (Table 6, Fig. 4).

\section{Correlation between number of arousals and frontal beta and SOD of TST}

Spearman's correlation was performed on frontal beta and the SOD due to the non-normality of the data. The number of arousals was correlated with the SOD of TST $(\mathrm{r}=0.532, P=0.023)$ in the IWHW group (Table 7 , Fig. 5).

\section{Correlation between number of arousals and central beta, SOD of TST}

Spearman's correlation was performed on the SOD of TSTS due to the non-normality of the data. The number of arousals was correlated with the SOD of TST $(\mathrm{r}=0.532, P=0.023)$ in the IWHW group (Table 8$)$.

\section{Discussion}

To the best of our knowledge, this is the first study that has investigated the absolute and relative spectral power of young adult patients (18-40 years old) with subtypes of subjective insomnia. Here, we categorized insomnia patients into IWLM and IWHM groups to maximize the difference in the SOD. Overall, compared to the GS 
Table 6 Correlation between relative EEG spectral power and SOD of TST

\begin{tabular}{lcc}
\hline Variables & \multicolumn{2}{l}{ SOD of TST } \\
\cline { 2 - 3 } & $\mathbf{r}$ & $\boldsymbol{P}$ \\
\hline Frontal derivation & & \\
Delta (1-4 Hz) & -0.248 & 0.092 \\
Theta (4-8 Hz & 0.127 & 0.397 \\
Alpha (8-12 Hz) & 0.158 & 0.290 \\
Sigma (8-12 Hz) & 0.124 & 0.405 \\
Beta (16-32 Hz) & 0.313 & 0.032 \\
Beta/Delta & 0.363 & 0.012 \\
Beta/Theta & 0.208 & 0.161 \\
Alpha/Delta & 0.173 & 0.245 \\
Alpha/Theta & 0.182 & 0.221 \\
Central derivation & & \\
Delta (1-4 Hz) & -0.268 & 0.069 \\
Theta (4-8 Hz & -0.072 & 0.631 \\
Alpha (8-12 Hz) & 0.247 & 0.094 \\
Sigma (8-12 Hz) & 0.207 & 0.163 \\
Beta (16-32 Hz) & 0.232 & 0.116 \\
Beta/Delta & 0.249 & 0.091 \\
Beta/Theta & 0.1888 & 0.205 \\
Alpha/Delta & 0.256 & 0.082 \\
Alpha/Theta & 0.169 & 0.257 \\
\hline SOD: Subjective-objective sleep discrepancy: TST:total sleep time &
\end{tabular}

SOD: Subjective-objective sleep discrepancy; TST: total sleep time

group, patients with IWHM exhibited an increase in the absolute power and relative beta/delta ratio in the frontal region during sleep. Moreover, the SOD of TST was positively correlated with the absolute power and relative beta/delta ratio in the same frontal region. However, no significant difference was observed in the EEG power or beta/delta ratio in the central region and no significant difference in the above parameters was observed between the IWHM and IWLM groups.

Beta power is generally considered an indicator of cortical arousal. It has been shown that beta activity in PI patients is higher than that in GS [14-17], which suggests that patients with subjective insomnia may experience enhanced sensory processing during sleep. In fact, this phenomenon may render them highly responsive and sensitive to external sounds and in turn may also lead to the mistaken perception of their sleep as wakefulness [13]. Based on prior studies, sigma activity (sleep spindle) represents a marker of sleep stability, especially against noises [15]. Therefore, sigma activity may be able to distort the transmission of auditory information to the cortex during sleep [28]. A study from Spiegelhalder et al. [15] proposed the concept of simultaneous activation of wake-promoting and sleep-protecting neural activity patterns in PI. However, a meta-analysis showed that sigma increase during NREM sleeps in PI exhibited moderate and high heterogeneity in the dispersion of effect sizes [18]. Here, we found that both the IWHM and IWLM groups exhibited no increase in absolute and relative power of all frequency bands in the central and frontal regions. Our findings are similar to a previous EEG-based spectral investigation by Buysse et al. [29] that failed to find significant differences in the frequency band activity between insomnia types and GS during NREM sleep. Nevertheless, unlike other previous work, we failed to observe lower delta NREM EEG activity, or greater alpha, theta, sigma, beta NREM EEG activity in patients with insomnia. This discrepancy was unclear but could be influenced by the difference in the age, frequency band definitions and diagnostic criteria of IWHM and IWLW patients in the two studies. To our knowledge, there has been little research on the relationship between age and power spectra, leading to dissimilar results. For example, Krystal et al. [19] reported that older age (40-80 years-old) was associated with significantly lower sigma $(12.5-16 \mathrm{~Hz})$ relative power during NREM in insomnia patients. Svetnik et al. [23] demonstrated that the power of the delta, theta and sigma bands significantly decreased with age whereas the slope in the alpha, beta and gamma bands did not. Therefore, age may be a potential influencing factor.

Insomnia is associated with poorer cognitive performance both generally and across multiple specific cognitive domains, especially in terms of a decline of working memory and executive ability $[30,31]$. A longer course of insomnia generally leads to a poorer cognitive impairment, which manifests as slower EEG frequency, a higher proportion of alpha and beta band power, and a lower proportion of theta and delta band power. It will be more conducive if the relationship between age and power spectrum could be studied in combination with the course of disease. To our knowledge, PSA studies categorizing insomnia into subtypes are limited. Some studies determined if the PSG was normal as a basis for judging subjective and objective insomnia [19], which may have led to the inclusion of patients with different subtypes of insomnia. Other studies have also explored SOD of SOL. In our research, insomnia patients were further categorized into two subgroups based on their SOD of TST. IWLM individuals exhibited a SOD $<60 \mathrm{~min}$ in TST whereas IWHM individuals exhibited a SOD $>120 \mathrm{~min}$ in TST. In our study, the PSG of patients with IWHM and IWLW was normal and the PSQI was higher than GS participants. This meant that all patients were of subjective insomnia, but that the degree of SOD was different. SOD of IWHW insomnia patients was greater than 120 min in TST, while that of IWLW insomnia patients 


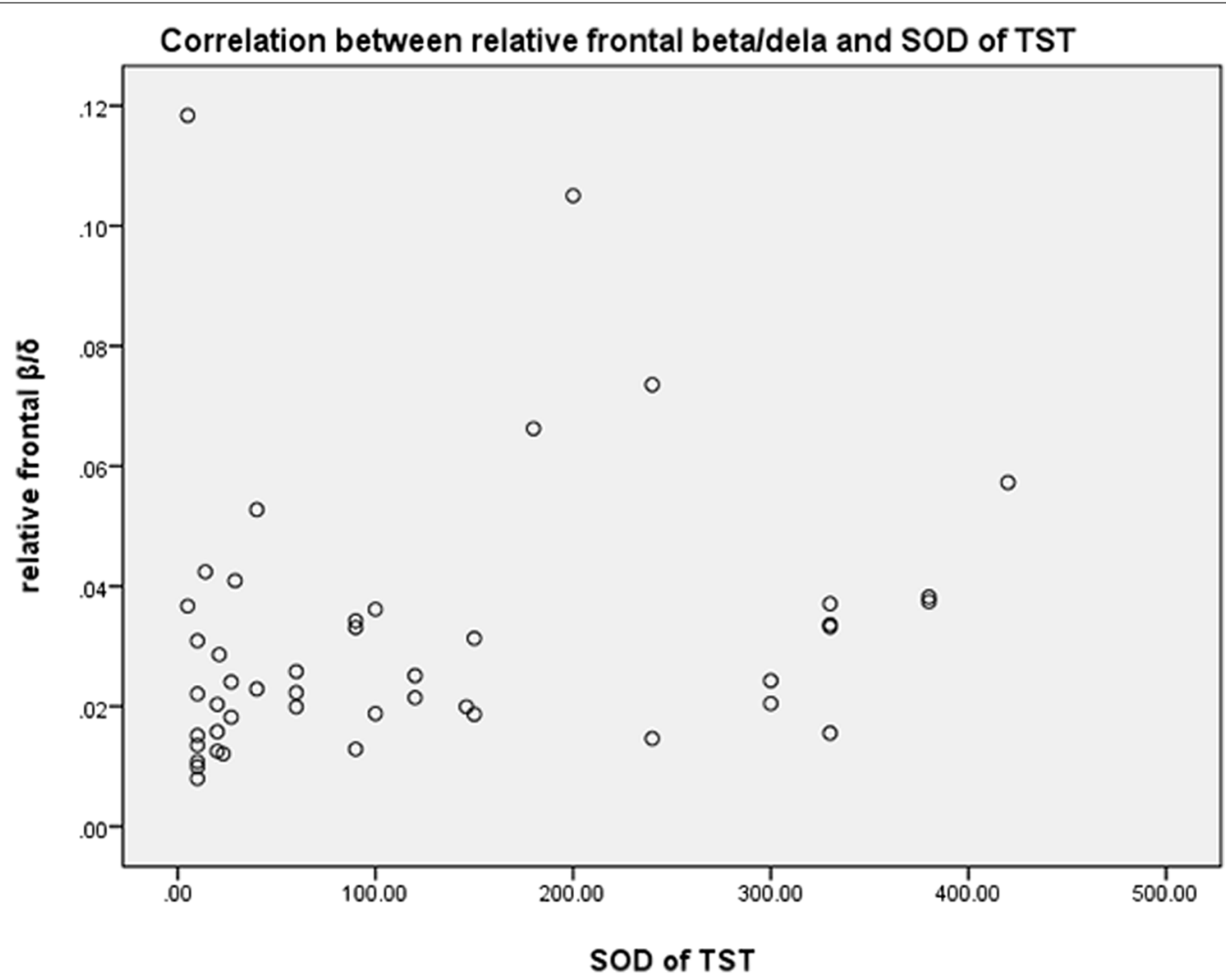

Fig. 4 Correlation between relative frontal beta/dela spectral power and SOD of TST. SOD: Subjective-objective sleep discrepancy; TST: total sleep time

was less than 120 min. In addition, patients with subjective insomnia met persistent PI criteria and had a normal

Table 7 Correlation between the number of arousals and frontal beta and SOD of TST

\begin{tabular}{lll}
\hline Variables & \multicolumn{2}{l}{ Number of arousals } \\
\cline { 2 - 3 } & $\mathbf{r}$ & $\boldsymbol{P}$ \\
\hline GS $(\mathrm{n}=10)$ & -0.049 & \\
Absolute beta & -0.482 & 0.894 \\
Relative beta & -0.028 & 0.159 \\
SOD of TST & & 0.938 \\
IWHM ( $\mathrm{n}=18)$ & 0.271 & \\
Absolute beta & 0.253 & 0.277 \\
Relative beta & 0.532 & 0.311 \\
SOD of TST & & 0.023 \\
IWLM (n=19) & 0.395 & \\
Absolute beta & 0.167 & 0.094 \\
Relative beta & -0.190 & 0.495 \\
SOD of TST & 0.435 \\
\hline
\end{tabular}

SOD: Subjective-objective sleep discrepancy single night PSG in Krystal's paper, which did not clearly define SOD.

Recently, the ratio of high-frequency to low-frequency EEG power has been recognized as a novel indicator of cortical arousal. Furthermore, individuals with a higher ratio of this sort may have more sleeping difficulties. Meric et al. [32] found that Psyl patients exhibited an increased beta/delta ratio in the temporal lobe during the sleep onset period (SOP). Some studies have also reported that delta EEG activity is decreased in PI patients in the temporal and central brain regions during the SOP [33, 34]. Thus, such an activity index (beta/delta ratio) may be a more appropriate indicator of cortical arousal in insomnia patients [17, 32, 35]. In the current study, IWHM patients showed increased absolute power and relative beta/delta ratio in the frontal region compared with the GS group, suggesting hyperarousal in the frontal portion of the brain.

SOD in insomnia has been shown to arise due to several possible mechanisms, which mainly focus on sensory perception, emotion and cognition [9]. Various characteristics of insomnia patients support these concepts, such as: (1) insomniacs will judge PSG measured 


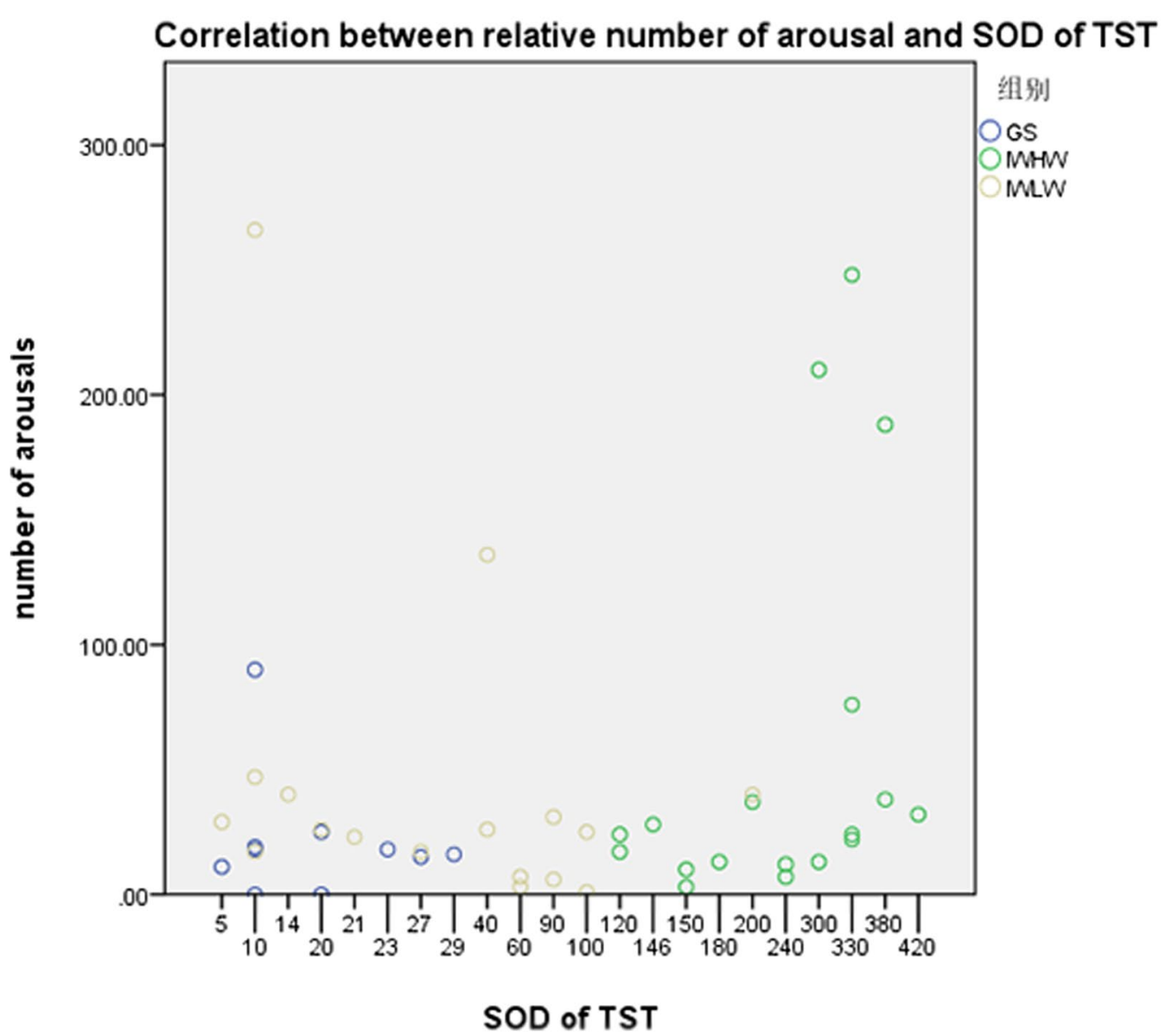

Fig. 5 Correlation between the number of arousals and SOD of TST. SOD, Subjective-objective sleep discrepancy; TST: total sleep time; GS: good sleeper; IWLM: insomnias with a low mismatch; IWHM: insomnias with a high mismatch

Table 8 Correlation between the number of arousals and central beta and SOD of TST

\begin{tabular}{lcc}
\hline Variables & \multicolumn{2}{l}{ Number of arousals } \\
\cline { 2 - 3 } & $\mathbf{r}$ & $\boldsymbol{P}$ \\
\hline GS $(\mathrm{n}=10)$ & 0.055 & \\
Absolute beta & -0.085 & 0.880 \\
Relative beta & -0.028 & 0.815 \\
SOD of TST & & 0.938 \\
IWHM ( $\mathrm{n}=18)$ & 0.413 & \\
Absolute beta & -0.030 & 0.089 \\
Relative beta & 0.532 & 0.906 \\
SOD of TST & & 0.023 \\
IWLM (n=19) & 0.173 & \\
Absolute beta & -0.155 & 0.479 \\
Relative beta & 0.190 & 0.527 \\
SOD of TST & 0.435 \\
\hline
\end{tabular}

SOD: Subjective-objective sleep discrepancy sleep as wakefulness; (2) insomniacs have anxiety and selective attention toward sleep-related threats. The possibility that anxiety serves to trigger the misperception of sleep is drawn from the robust finding in time perception literature in that time is perceived as longer when the number of units of information processed per unit of time increases. Other characteristics of insomnia patients include: (3) patients may simply be poor estimators of time; and (4) insomniacs' assessment of sleep quality is influenced by a memory bias that is influenced by current symptoms and emotions, a confirmation bias/ belief bias or a recall bias linked to intensity. In many other papers, central regions, mainly involving in sensory perception, are considered good representations of the whole brain activity (from EEG) and have been widely used in PSA. Frontal lobes are also related to emotion, cognition, and behavioral management, which is connected with the mechanism of SOD. Therefore, it is necessary to assess frontal regions. Unfortunately, cortical activation at sites other than central areas, such as 
frontal regions, has been poorly explored. In our study, a higher beta/delta ratio was only observed in frontal regions in the IWHW group when comparison to GS group. This result seems to suggest that high cortical arousal occurs in the frontal lobe and not just in the central region.

We further showed that the SOD of TST was associated with the absolute and relative NREM beta/delta ratio $(r=0.363)$ and relative beta power $(r=0.313)$ in the frontal area. All in all, these results indicate that a higher the beta/delta ratio and beta power during NREM sleep may be an underestimation of TST. Our results are similar to the findings by Perlis et al. [14] that showed a moderate correlation between the SOD of TST and NREM beta activity $(14-35 \mathrm{~Hz})(\mathrm{r}=-0.46)$. The underestimation of TST may be explained by the insertion of high frequency EEG into low frequency EEG, which has been shown to enhance the information processing ability and to degrade sleep quality [36].

To the best of our knowledge, few studies have reported the correlation between the SOD of TST and the number of arousals. Results from our study showed that the number of arousals was correlated with the SOD of TST in the IWHW group, suggesting that frequent awakenings that lead to sleep fragmentation may in turn lead to poor perception of insomnia. This is similar to the previous study by Choi et al. [37] that showed that sleep perception was negatively related to the PSG arousal index.

There are various limitations to our study that must be noted. First, only one PSG recording was performed in each participant and thus, the results might be biased by the "first night" effect. Secondly, insomnia patients were categorized into the IWHM and IWLM groups based only on TST. The percentage of SWS should also be considered in future investigations. Lastly, the sample size in this work was relatively small. Future studies with larger sample sizes are needed to further elucidate the neurophysiological mechanisms about the SOD.

\section{Conclusion}

In conclusion, the present study suggests that IWHM insomnia in young adults is associated with increased absolute power and relative beta/delta ratio in the frontal brain region. However, there was no significant difference observed between the IWHM and IWLM groups. Furthermore, the SOD of TST was associated with the frontal NREM beta/delta ratio. This indicate that the increased beta/delta ratio is a characteristic of sleep misperception. Finally, it is necessary to look at both frontal and central regions when investigating the mechanism of insomnia.

\section{Acknowledgements}

We want to thank Chen Wang and Yujiao Sun who helped in the statistical analysis.

\section{Authors' contributions}

BX: data analysis and interpretation, manuscript drafting/revision. QC: major role in data acquisition. RM: manuscript revision. HL: study supervision, critical revision of the manuscript. JH: data collection, manuscript revision. ZY: study concept and design, critical revision of the manuscript. All authors read and approved the final manuscript.

\section{Funding}

This study was supported by 2014 Science and Technology Projects of Guangdong Province [2014A020212557]; Zhimin Yang Guangdong famous

Chinese Medicine Inheritance studio construction project [Guangdong Chinese Medicine (2020) 1].

Availability of data and materials

The datasets used and/or analysed during the current study are available from the corresponding author on request.

\section{Declarations}

\section{Ethics approval and consent to participate}

This clinical trial was approved by the Ethics Committee of the Guangdong Provincial Hospital of Chinese Medicine (number: B3016-075) and performed in accordance with the World Medical Association Declaration of Helsinki.

This study was registered on http://www.chictr.org/up (registration number: chiCTR-COC-16008530). Informed consent was obtained from all subjects prior to participation.

\section{Consent for publication}

Not applicable.

\section{Competing interests}

The authors declare that they have no competing interests.

\section{Author details}

${ }^{1}$ Department of Fangcun Sleep-Disorder, the Second Clinical College of Guangzhou University of Chinese Medicine (Guangdong Provincial Hopsital of Chinese Medicine), Guangzhou 510120, China. ${ }^{2}$ Applicants for Doctoral Degree with an Equivalent Educational Level in Guangzhou University of Chinese Medicine, Guangzhou 510006, China. ${ }^{3} 111$ Dade Road, Yuexiu District, Guangzhou 510120, China.

Received: 18 March 2021 Accepted: 17 January 2022

Published online: 24 January 2022

\section{References}

1. Buysse DJ. Insomnia. JAMA. 2013;309(7):706-16. https://doi.org/10.1001/ jama.2013.193.

2. Baglioni C, Battagliese G, Feige B, Spiegelhalder K, Nissen C, Voderholzer U, Lombardo C, Riemann D. Insomnia as a predictor of depression: a meta-analytic evaluation of longitudinal epidemiological studies. J Affect Disord. 2011;135(1-3):10-9. https://doi.org/10.1016/j.jad.2011.01.011.

3. Laugsand LE, Vatten LJ, Platou C, Janszky I. Insomnia and the risk of acute myocardial infarction. Circulation. 2011;124(19):2073-81. https://doi.org/ 10.1161/CIRCULATIONAHA.111.025858.

4. Riemann D, Spiegelhalder K, Espie C, Pollmacher T, Leger D, Bassetti C, van Someren E. Chronic insomnia: clinical and research challenges-an agenda. Pharmacopsychiatry. 2010;44(1):1-14. https://doi.org/10.1055/s0030-1267978.

5. Hillman D, Mitchell S, Streatfeild J, Burns C, Bruck D, Pezzullo L. The economic cost of inadequate sleep. Sleep. 2018. https://doi.org/10.1093/ sleep/zsy083.

6. Sivertsen B, Lallukka T, Salo P. The economic burden of insomnia at the workplace. An opportunity and time for intervention? Sleep. 2011;34(9):1151-2. https://doi.org/10.5665/SLEEP.1224. 
7. Hillman DR, Murphy AS, Pezzullo L. The economic cost of sleep disorders. Sleep. 2006;29(3):299-305. https://doi.org/10.1093/sleep/zsy083.

8. Ihler HM, Meyrel M, Hennion V, Maruani J, Gross G, Geoffroy PA, Lagerberg TV, Melle I, Bellivier F, Scott J, Etain B. Misperception of sleep in bipolar disorder: an exploratory study using questionnaire versus actigraphy. Int $J$ Bipolar Disord. 2020;8(1):34. https://doi.org/10.1186/s40345-020-00198-x.

9. Harvey AG, Tang NKY. (Mis)perception of sleep in insomnia: a puzzle and a resolution. Psychol Bull. 2012;138(1):77-101. https://doi.org/10.1037/ a0025730.

10. Tang NKY, Harvey AG. Time estimation ability and distorted perception of sleep in insomnia. Behav Sleep Med. 2005;3(3):134-50. https://doi.org/10. 1207/s15402010bsm0303_2.

11. Castelnovo A, Ferri R, Punjabi NM, Castronovo V, Garbazza C, Zucconi M, Ferini-Strambi L, Manconi M. The paradox of paradoxical insomnia: a theoretical review towards a unifying evidence-based definition. Sleep Med Rev. 2019:44:70-82. https://doi.org/10.1016/j.smrv.2018.12.007.

12. Marzano C, Ferrara M, Sforza E, De Gennaro L. Quantitative electroencephalogram (EEG) in insomnia: a new window on pathophysiological mechanisms. Curr Pharm Des. 2008;14(32):3446-55. https://doi.org/10. 2174/138161208786549326.

13. Riemann D, Spiegelhalder K, Feige B, Voderholzer U, Berger M, Perlis M, Nissen $C$. The hyperarousal model of insomnia: a review of the concept and its evidence. Sleep Med Rev. 2010;14(1):19-31. https://doi.org/10, 1016/j.smrv.2009.04.002

14. Perlis ML, Smith MT, Andrews PJ, Orff H, Giles DE. Beta/Gamma EEG activity in patients with primary and secondary insomnia and good sleeper controls. Sleep. 2001;24(1):110-7. https://doi.org/10.1093/sleep/24.1.110.

15. Spiegelhalder K, Regen W, Feige B, Holz J, Piosczyk H, Baglioni C, Riemann $D$, Nissen C. Increased EEG sigma and beta power during NREM sleep in primary insomnia. Biol Psychol. 2012;91(3):329-33. https://doi.org/10. 1016/j.biopsycho.2012.08.009

16. Kwan Y, Baek C, Chung S, Kim TH, Choi S. Resting-state quantitative EEG characteristics of insomniac patients with depression. Int J Psychopyhsiol. 2018;124:26-32. https://doi.org/10.1016/j.jpsycho.2018.01.005.

17. Riedner BA, Goldstein MR, Plante DT, Rumble ME, Ferrarelli F, Tononi G, Benca RM. Regional patterns of elevated alpha and high-frequency electroencephalographic activity during nonrapid eye movement sleep in chronic insomnia: a pilot study. Sleep. 2016;39(4):801-12. https://doi. org/10.5665/sleep.5632.

18. Zhao W, Van Someren EJW, Li C, Chen X, Gui W, Tian Y, Liu Y, Lei X. EEG spectral analysis in insomnia disorder: a systematic review and metaanalysis. Sleep Med Rev. 2021;59: 101457. https://doi.org/10.1016/J.SMRV. 2021.101457.

19. Krystal AD, Edinger JD, Wohlgemuth WK, Marsh GR. NREM sleep EEG frequency spectral correlates of sleep complaints in primary insomnia subtypes. Sleep. 2002;25(6):630-40.

20. St-Jean G, Turcotte I, Pérusse AD, Bastien CH. REM and NREM power spectral analysis on two consecutive nights in psychophysiological and paradoxical insomnia sufferers. Int J Psychopyhsiol. 2013;89(2):181-94. https://doi.org/10.1016/j.ijpsycho.2013.06.004.

21. Lecci S, Cataldi J, Betta M, Bernardi G, Heinzer R, Siclari F. EEG changes associated with subjective under- and overestimation of sleep duration. Sleep. 2020. https://doi.org/10.1093/sleep/zsaa094.

22. Chellappa SL, Munch M, Knoblauch V, Cajochen C. Age effects on spectral electroencephalogram activity prior to dream recall. J Sleep Res. 2012;21(3):247-56. https://doi.org/10.1111/j.1365-2869.2011.00947.x.

23. Svetnik V, Snyder ES, Ma J, Tao P, Lines C, Herring WJ. EEG spectral analysis of NREM sleep in a large sample of patients with insomnia and good sleepers: effects of age, sex and part of the night. J Sleep Res. 2017;26(1):92-104. https://doi.org/10.1111/jsr.12448.

24. Dijk D, Duffy JF, Riel E, Shanahan TL, Czeisler CA. Ageing and the circadian and homeostatic regulation of human sleep during forced desynchrony of rest, melatonin and temperature rhythms. J Physiol. 1999;516(2):61127. https://doi.org/10.1111/j.1469-7793.1999.0611v.x.

25. Xianchen L, Tang M, Hu L, Wang A, Wu H. Reliability and validity of the Pitts-burgh sleep quality index-Chinese version. Chin J Psychiatry. 1996;29:103-7.

26. Bonicatto S, Dew MA, Soria JJ, Seghezzo ME. Validity and reliability of Symptom Checklist '90 (SCL90) in an Argentine population sample. Soc Psychiatry Psychiatr Epidemiol. 1997;32(6):332-8. https://doi.org/10.1007/ BF00805438.
27. Berry RB, Quan SF, Abreu AR, Bibbs M, DelRosso L, Harding SM, Mao MM, Plante DT, Pressman MR, Troester MM, Vaughn BV. The AASM Manual for the Scoring of Sleep and Associated Events. Version 2.5. Darien, IL, USA: American Academy of Sleep Medicine, 2018.

28. Lüthi A. Sleep spindles where they come from, what they do. Neuroscientist. 2014;20(3):243-56. https://doi.org/10.1177/1073858413500854.

29. Buysse DJ, Germain A, Hall ML, Moul DE, Nofzinger EA, Begley A, Ehlers CL, Thompson W, Kupfer DJ. EEG spectral analysis in primary insomnia: NREM period effects and sex differences. Sleep. 2008;31(12):1673-82. https://doi.org/10.5664/jcsm.6280.

30. Wardle-Pinkston S, Slavish DC, Taylor DJ. Insomnia and cognitive performance: a systematic review and meta-analysis. Sleep Med Rev. 2019;48: 101205. https://doi.org/10.1016/.smrv.2019.07.008.

31. De Bruin EJ, van Run C, Staaks J, Meijer AM. Effects of sleep manipulation on cognitive functioning of adolescents: a systematic review. Sleep Med Rev. 2017;32:45-57. https://doi.org/10.1016/j.smrv.2016.02. 006.

32. Merica H, Gaillard JM. The EEG of the sleep onset period in insomnia: a discriminant analysis. Physiol Behav. 1992;52(2):199. https://doi.org/10. 1016/0031-9384(92)90258-4.

33. Lamarche $\mathrm{CH}$, Ogilvie RD. Electrophysiological changes during the sleep onset period of psychophysiological insomniacs, psychiatric insomniacs, and normal sleepers. Sleep. 1997;20(9):724-33.

34. Cervena K, Espa F, Perogamvros L, Perrig S, Merica H, Ibanez V. Spectral analysis of the sleep onset period in primary insomnia. Clin Neurophysiol. 2014;125(5):979-87. https://doi.org/10.1016/j.clinph.2013.10.010.

35. Ferri R, Cosentino Fll, Manconi M, Rundo F, Bruni O, Zucconi M. Increased electroencephalographic high frequencies during the sleep onset period in patients with restless legs syndrome. Sleep. 2014;37(8):1375-81. https://doi.org/10.5665/sleep.3934.

36. Maes J, Verbraecken J, Willemen M, De Volder I, van Gastel A. Sleep misperception, EEG characteristics and autonomic nervous system activity in primary insomnia: a retrospective study on polysomnographic data. Int J Psychophysiol. 2014;91(3):163-71. https://doi.org/10.1016/j.jpsy cho.2013.10.012.

37. Choi SJ, Suh S, Ong J, Joo EY. Sleep misperception in chronic insomnia patients with obstructive sleep apnea syndrome: implications for clinical assessment. J Clin Sleep Med. 2016;12(11):1517-25. https://doi.org/ 10.5664/jcsm.6280.

\section{Publisher's Note}

Springer Nature remains neutral with regard to jurisdictional claims in published maps and institutional affiliations.

Ready to submit your research? Choose BMC and benefit from:

- fast, convenient online submission

- thorough peer review by experienced researchers in your field

- rapid publication on acceptance

- support for research data, including large and complex data types

- gold Open Access which fosters wider collaboration and increased citations

- maximum visibility for your research: over $100 \mathrm{M}$ website views per year

At BMC, research is always in progress.

Learn more biomedcentral.com/submissions 